\title{
TWO EXCEPTIONAL 3-ADIC AFFINE BUILDINGS
}

\section{INTRODUCTION}

This note contains constructions of some discrete chamber-transitive automorphism groups of affine buildings for certain 5- and 6-dimensional orthogonal groups over the field $\mathbb{Q}_{3}$ of 3-adic numbers. Such groups are very rare. Similar constructions can be found in [4], [7], [9] and [17], and the groups obtained by such constructions have been classified in [6] in the case of Witt index $\geqslant 2$ in arbitrary dimension for an arbitrary local field.

One motivation for such constructions arises from the fact that, upon passage modulo suitable normal subgroups, they produce finite building-like geometries having chamber-transitive automorphism groups. Such geometries have, in turn, been characterized group-theoretically (cf. [8], [11], [12], [13] and their references).

We have described the examples in a very concrete manner as arithmetic groups. Consequently, all finite homomorphic images can be immediately obtained, in view of the results in [5].

\section{AFFINE BUILDINGS OF 5-AND 6-DIMENSIONAL ORTHOGONAL GROUPS}

We will need descriptions of two affine buildings. These are based on [3] and [14].

Consider a vector space $V$ over $\mathbb{Q}_{3}$. Let $\mathbb{Z}_{3}$ denote the ring of 3-adic integers.

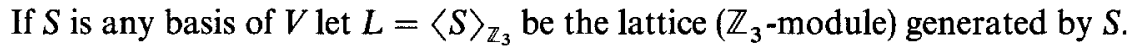
Write $[L]=\left\{a L \mid 0 \neq a \in \mathbb{Q}_{3}\right\}$. Note that all members of a lattice-class $[L]$ have the same stabilizer in GL(V).

Now assume that $V$ has dimension 6 , with basis $e_{1}, e_{2}, f_{1}, f_{2}, u_{5}, u_{6}$, and is equipped with the inner product $f=\left(\right.$, ) defined by $\left(e_{i}, e_{j}\right)=\left(f_{i}, f_{j}\right)=0$, $\left(e_{i}, f_{j}\right)=\delta_{i j},\left(e_{i}, u_{k}\right)=\left(f_{i}, u_{k}\right)=\left(u_{5}, u_{6}\right)=0,\left(u_{5}, u_{5}\right)=1$ and $\left(u_{6}, u_{6}\right)=3$ for all $i, j, k$. Then the affine building of the corresponding orthogonal group $\mathrm{O}\left(f, \mathbb{Q}_{3}\right)$ is the simplicial complex $\Delta$ whose vertices (of respective types 0,1 and 2) are the

* This research was supported in part by NSF Grants DMS 87-01794 and INT 85-12644.

Geometriae Dedicata 33: 1-11, 1990.

(C) 1990 Kluwer Academic Publishers. Printed in the Netherlands. 
lattice-classes $\left[\Lambda_{0}\right]^{g},\left[\Lambda_{1}\right]^{g},\left[\Lambda_{2}\right]^{g}, g \in \mathrm{O}\left(f, \mathbb{Q}_{3}\right)$, where $\Lambda_{i}$ is defined by

$$
\begin{aligned}
& \Lambda_{0}=\left\langle e_{1}, e_{2}, f_{1}, f_{2}, u_{5}, u_{6}\right\rangle_{\mathbb{Z}_{3}} \\
& \Lambda_{1}=\left\langle e_{1} / 3, e_{2}, f_{1}, f_{2}, u_{5}, u_{6} / 3\right\rangle_{\mathbb{Z}_{3}} \\
& \Lambda_{2}=\left\langle e_{1} / 3, e_{2} / 3, f_{1}, f_{2}, u_{5}, u_{6} / 3\right\rangle_{\mathbb{Z}_{3}} .
\end{aligned}
$$

One chamber (i.e., maximal simplex) of $\Delta$ is $\left\{\left[\Lambda_{0}\right],\left[\Lambda_{1}\right],\left[\Lambda_{2}\right]\right\}$, with all others obtained by applying elements of $\mathrm{O}\left(f, \mathbb{Q}_{3}\right)$. Note that the transformation $\theta$ taking

$$
\begin{aligned}
& e_{1} \rightarrow 3 f_{2} \rightarrow-3 e_{1} \\
& e_{2} \rightarrow-3 f_{1} \rightarrow-3 e_{2} \\
& u_{5} \rightarrow u_{6} \rightarrow-3 u_{5}
\end{aligned}
$$

sends $\Lambda_{1}$ to its dual lattice

$$
\Lambda_{1}^{\#}:=\left\{x \in V \mid\left(x, \Lambda_{1}\right) \subseteq \mathbb{Z}_{3}\right\}=\left\langle e_{1}, e_{2}, 3 f_{1}, f_{2}, u_{5}, u_{6}\right\rangle_{\mathbb{Z}_{3}},
$$

and sends $\Lambda_{2} \rightarrow \Lambda_{0} \rightarrow 3 \Lambda_{2}$. Here, $\theta$ satisfies $\left(u^{\theta}, v^{\theta}\right)=3(u, v)$ for all $u, v \in V$, and $\theta^{2}=-3$. Note that the stabilizer of $\left[\Lambda_{1}\right]$ in $\mathrm{O}\left(f, \mathbb{Q}_{3}\right)$ coincides with that of $\left[\Lambda_{1}^{\#}\right]$.

The building $\Delta$ has the diagram ; cf. [14]). This has, among others, the following meaning. $\operatorname{Star}\left(\left[\Lambda_{0}\right]\right)$, the star of $\left[\Lambda_{0}\right]$, is the finite building of the orthogonal group $\mathrm{O}(5,3)$. Namely, the bilinear form $\left(u+3 \Lambda_{0}, v+3 \Lambda_{0}\right) \bmod 3$ induced on the GF(3)-space $\bar{\Lambda}_{0}:=\Lambda_{0} / 3 \Lambda_{0}$ has radical $\bar{\Lambda}_{0}^{\perp}$ generated by $u_{6}+3 \Lambda_{0}$. Moreover,

(\#) If $\Lambda$ is a lattice in $V$, then $[\Lambda]$ represents a vertex of $\Delta_{6}$ adjacent to $\left[\Lambda_{0}\right]$ if and only if some member of $[\Lambda]$, say $\Lambda$ itself, satisfies the following conditions: $3 \Lambda_{0} \subset \Lambda \subset \Lambda_{0}, \Lambda / 3 \Lambda_{0} \supset \bar{\Lambda}_{0}^{\perp}$, and $\Lambda / 3 \Lambda_{0}$ is totally singular.

(In fact, $3 \Lambda_{1} / 3 \Lambda_{0}$ and $3 \Lambda_{2} / 3 \Lambda_{0}$ are the totally singular subspaces spanned by $e_{1}+3 \Lambda_{0}, u_{6}+3 \Lambda_{0}$ and $e_{1}+3 \Lambda_{0}, e_{2}+3 \Lambda_{0}, u_{6}+3 \Lambda_{0}$, respectively.) Similarly, $\operatorname{Star}\left(\left[\Lambda_{1}\right]\right)$ and $\operatorname{Star}\left(\left[\Lambda_{2}\right]\right)$ are a generalized digon and the $O(5,3)$ building, respectively. $\left(\right.$ In fact, $\operatorname{Star}\left(\left[\Lambda_{2}\right]\right)=\operatorname{Star}\left(\left[\Lambda_{0}\right]\right)^{\theta}$.)

Clearly, $\mathrm{O}\left(f, \mathbb{Q}_{3}\right)$ acts on $\Delta_{6}$ with induced automorphism group $\operatorname{PO}\left(f, \mathbb{Q}_{3}\right)=\mathrm{O}\left(f, \mathbb{Q}_{3}\right) /\langle-1\rangle$.

The second building $\Delta^{\prime}$ we will consider is that of the commutator subgroup $\Omega\left(f^{\prime}, \mathbb{Q}_{3}\right)$ of the orthogonal group $\mathrm{O}\left(f^{\prime}, \mathbb{Q}_{3}\right)$ of the form $f^{\prime}$ obtained by restricting $f$ to the orthogonal complement $V^{\prime}:=\left\langle e_{1}, e_{2}, f_{1}, f_{2}, u_{5}\right\rangle$ of $u_{6}$. 
This building is defined as above, this time using the three lattices

$$
\begin{aligned}
& \Lambda_{0}^{\prime}=\left\langle e_{1}, e_{2}, f_{1}, f_{2}, u_{5}\right\rangle_{\mathbb{Z}_{3}} \\
& \Lambda_{1}^{\prime}=\left\langle e_{1} / 3, e_{2} / 3, f_{1}, f_{2}, u_{5}\right\rangle_{\mathbb{Z}_{3}} \\
& \Lambda_{2}^{\prime}=\left\langle e_{1} / 3, e_{2}, 3 f_{1}, f_{2}, u_{5}\right\rangle_{\mathbb{Z}_{3}} .
\end{aligned}
$$

The diagram is respective stars the $O(5,3)$ building, a generalized digon, and the $O(5,3)$ building. This time $\mathrm{O}\left(f^{\prime}, \mathbb{Q}_{3}\right)$ is not type-preserving: the reflection in $\left(e_{1}-3 f_{1}\right)^{\perp}$ fixes $\left[\Lambda_{1}^{\prime}\right]$ and interchanges $\left[\Lambda_{0}^{\prime}\right]$ and $\left[\Lambda_{2}^{\prime}\right]$.

\section{THE 6-DIMENSIONAL DISCRETE GROUPS}

Let $V_{6}$ be the 6-dimensional vector space $\mathbb{Q}^{6}$, with standard basis $u_{1}, u_{2}, u_{3}$, $u_{4}, u_{5}, u_{6}$, equipped with the bilinear form $f$ defined by $\left(u_{i}, u_{j}\right)=\delta_{i j}$ except that $\left(u_{6}, u_{6}\right)=3$. Let $V_{6}$ be embedded in $V=\mathbb{Q}_{3}^{6}$ in the natural manner, and let $f$ also denote the form induced on $V$.

We begin by relating the present notation to that of Section 2. Namely, let $\alpha \in \mathbb{Z}_{3}$ be the root of the equation $x^{2}+2=0$ such that $1+\alpha \notin 3 \mathbb{Z}_{3}$ (i.e., $1+\alpha$ is a unit) and $1-\alpha \in 3 \mathbb{Z}_{3}$. Let $e_{1}, e_{2}, f_{1}, f_{2}, u_{5}, u_{6}$ be the basis of $V$ defined by

$$
\begin{aligned}
& e_{1}=\frac{1}{2}(1+\alpha)\left(u_{1}+u_{2}+\alpha u_{3}\right) \\
& f_{1}=\frac{1}{2}(1+\alpha)^{-1}\left(u_{1}+u_{2}-\alpha u_{4}\right) \\
& e_{2}=\frac{1}{2}\left(u_{1}-u_{2}+\alpha u_{4}\right) \\
& f_{1}=\frac{1}{2}\left(u_{1}+u_{2}-\alpha u_{4}\right) .
\end{aligned}
$$

Then these basis vectors behave exactly as described in Section 2. In particular, $V$ has witt index 2 - while, clearly, $V_{6}$ is anisotropic.

Let $\Delta_{6}$ denote the building for the group $\Omega\left(f, \mathbb{Q}_{3}\right)$, and define $\left[\Lambda_{0}\right]$, $\left[\Lambda_{1}\right],\left[\Lambda_{2}\right]$ using the vectors $e_{1}, e_{2}, f_{1}, f_{2}, u_{5}, u_{6}$ just as in Section 2. The radical of $\bar{\Lambda}_{0}=\Lambda_{0} / 3 \Lambda_{0}$ is spanned by $u_{6}+3 \Lambda_{0}$.

PROPOSITION 1. $G_{6}:=\mathrm{O}\left(f, \mathbb{Q}_{3}\right) \cap \mathrm{GL}\left(6, \mathbb{Z}\left[\frac{1}{3}\right]\right)$ is discrete and chambertransitive on $\Delta_{6}$.

Proof. By a simple calculation, $\Lambda_{0}=\left\langle u_{1}, \ldots, u_{6}\right\rangle_{\mathbb{Z}_{3}}$. The stabilizer $\left(G_{6}\right)_{0}$ of the vertex $\left[\Lambda_{0}\right]$ consists of rational transformations preserving the $\mathbb{Z}$-lattice $\left\langle u_{1}, \ldots, u_{6}\right\rangle_{\mathbb{Z}}$, and hence preserves its set $\left\{ \pm u_{1}, \ldots, \pm u_{5}\right\}$ of squared length 1 vectors, so that $\left(G_{6}\right)_{0}$ is a monomial group of the form $2^{6} S_{5}$. In particular, this group induces a chamber-transitive group on $\operatorname{Star}\left(\left[\Lambda_{0}\right]\right)$. 
Now consider the transformation $\theta$ defined by the matrix

$$
\left(\begin{array}{rrrrrr}
0 & -1 & 1 & -1 & 0 & 0 \\
1 & 0 & -1 & -1 & 0 & 0 \\
-1 & 1 & 0 & -1 & 0 & 0 \\
1 & 1 & 1 & 0 & 0 & 0 \\
0 & 0 & 0 & 0 & 0 & 1 \\
0 & 0 & 0 & 0 & -3 & 0
\end{array}\right)
$$

Then $\left(u^{\theta}, v^{\theta}\right)=3(u, v)$ for all $u, v \in V$, and $\theta^{2}=-3$. It follows easily that $\left[\Lambda_{0}^{\theta}\right]$ has the properties in (\#) (cf. Section 2; in fact, $\Lambda_{0}^{\theta} / 3 \Lambda_{0}=3 \Lambda_{2} / 3 \Lambda_{0}$ is the totally singular 3-space spanned by $(1,1,1,0,0,0)+3 \Lambda_{0},(1,-1,0,1,0,0)+$ $3 \Lambda_{0}$ and $u_{6}+3 \Lambda_{0}$ ), and hence is a vertex of $\Delta_{6}$ of type 2 adjacent to $\left[\Lambda_{0}\right]$. Moreover, $\theta$ normalizes $G_{6}$ (since $\theta^{-1}$ has all entries in $\left.\mathbb{Z}\left[\frac{1}{3}\right]\right)$. Now $\left(G_{6}\right)_{0}^{\theta}$ is chamber-transitive on the star of $\left[\Lambda_{0}\right]^{\theta}$. Connectedness of $\Delta_{6}$ implies that the subgroup $\left\langle\left(G_{6}\right)_{0},\left(G_{6}\right)_{0}^{\theta}\right\rangle$ of $G_{6}$ is chamber-transitive on $\Delta_{6}$. Finally, $G_{6}$ is discrete since the stabilizer of any vertex is finite.

Since we now have two chamber-transitive automorphism groups $G_{6}$ and $\left\langle\left(G_{6}\right)_{0},\left(G_{6}\right)_{0}^{\theta}\right\rangle$ of $\Delta_{6}$ having the same vertex stabilizer, namely $\left(G_{6}\right)_{0}$, we deduce the

COROLLARY. The stabilizer $\left(G_{6}\right)_{0}$ of the vertex $\left[\Lambda_{0}\right]$ is $2{ }^{6} S_{5}$, and $G_{6}=$ $\left\langle\left(G_{6}\right)_{0},\left(G_{6}\right)_{0}^{\theta}\right\rangle$.

The vertices of $\Delta_{6}$ can all be represented by $\mathbb{Z}$-lattices, instead of by $\mathbb{Z}_{3}$-lattices as was done in the above proof and in Section 2. Namely, it is straightforward to check that $\left[\Lambda_{i}\right]=\left[\Sigma_{i} \otimes_{\mathbb{Z}} \mathbb{Z}_{3}\right]$ for $i=1,2,3$, where

$$
\Sigma_{0}:=\left\langle u_{1}, \ldots, u_{6}\right\rangle_{\mathbb{Z}}, \Sigma_{2}:=\Sigma_{0}^{\theta},
$$

and

$$
\begin{aligned}
\Sigma_{1}:= & \left\langle u_{1}+u_{2}-2 u_{3}, u_{2}+u_{3}-2 u_{1}\right\rangle_{\mathbb{Z}} \perp\left\langle 3 u_{4}, 3 u_{5}\right\rangle_{\mathbb{Z}} \perp \\
& \perp\left\langle u_{1}+u_{2}+u_{3}, u_{6}\right\rangle_{\mathbb{Z}} .
\end{aligned}
$$

Here, $\Sigma_{1} / 3 \Sigma_{0}$ is the totally singular 2-space of $\Sigma_{0} / 3 \Sigma_{0}$ spanned by $u_{1}+u_{2}+$ $u_{3}+3 \Sigma_{0}$ and $u_{6}+3 \Sigma_{0}$, while $\Sigma_{2} / 3 \Sigma_{0}$ is the totally singular 3-space spanned by $u_{1}+u_{2}+u_{3}+3 \Sigma_{0}, u_{1}-u_{2}+u_{4}+3 \Sigma_{0}$ and $u_{6}+3 \Sigma_{0}$. Moreover, from the description of $\Sigma_{1}$ it follows that the stabilizer $\left(G_{6}\right)_{1}$ of the vertex $\left[\Lambda_{1}\right]$ is $D_{12} \times D_{8} \times D_{8}$. Note that $\left(G_{6}\right)_{0} \cap\left(G_{6}\right)_{1}=S_{3} \times D_{8} \times 2$ and $\left(G_{6}\right)_{1}=\left[\left(G_{6}\right)_{0} \cap\right.$ 
$\left.\left(G_{6}\right)_{1}\right]\left[\left(G_{6}\right)_{2} \cap\left(G_{6}\right)_{1}\right]$. Since $\theta$ maps $(1 / 3) \Sigma_{1}$ onto the dual lattice $\Sigma_{1}^{\#}:=$ $\left\{v \in \mathbb{Q}^{6} \mid\left(v, \Sigma_{1}\right) \subseteq \mathbb{Z}\right\}$ of $\Sigma_{1}$ it normalizes $\left(G_{6}\right)_{1}$.

Next we turn to a new basis for $V_{6}$, as well as a new chamber-transitive automorphism group of $\Delta_{6}$.

Consider the following six vectors:

$$
\begin{aligned}
& z_{1}=\frac{1}{2}\left(-u_{1}-u_{2}-u_{3}-u_{4}+u_{5}+u_{6}\right) \\
& z_{2}=u_{4}+u_{5} \\
& z_{3}=u_{4}-u_{5} \\
& z_{4}=u_{1}-u_{4} \\
& z_{5}=u_{2}-u_{1} \\
& z_{6}=u_{3}-u_{2} .
\end{aligned}
$$

These vectors satisfy the relations of roots of a root system of type $E_{6}$ (cf. [2, p. 268]). Note that, with $\Lambda_{0}$ as before, we have $\Lambda_{0}=\left\langle z_{1}, z_{2}, z_{3}, z_{4}, z_{5}, z_{6}\right\rangle_{\mathbb{Z}_{3}}$.

Let $H_{6}$ denote the stabilizer of

$$
L:=\left\langle z_{1}, z_{2}, z_{3}, z_{4}, z_{5}, z_{6}\right\rangle_{\mathbb{Z}\left[\frac{1}{3}\right]}
$$

in $\mathrm{O}\left(f, \mathbb{Q}_{3}\right)$. (Alternatively, $H_{6}$ can be defined as $\mathrm{O}\left(e_{6}, \mathbb{Q}_{3}\right) \cap \mathrm{GL}\left(6, \mathbb{Z}\left[\frac{1}{3}\right]\right.$ ), where $e_{6}$ denotes the quadratic form for the $E_{6}$ root lattice, and matrices are written with respect to the $z_{i}$.)

PROPOSITION 2. $H_{6}$ is a discrete chamber-transitive automorphism group of $\Delta_{6}$.

Proof. This time, $\left(H_{6}\right)_{0}=\mathrm{W}\left(E_{6}\right) \times\langle-1\rangle$. The transformation $\theta$ sends $L$ to itself, and hence normalizes $H_{6}$. Thus, the argument in Proposition 1 can be repeated in the present situation.

COROLLARY. The stabilizer $\left(H_{6}\right)_{0}$ of the vertex $\left[\Lambda_{0}\right]$ is $\mathrm{W}\left(E_{6}\right) \times\langle-1\rangle$. Moreover, $H_{6}=\left\langle\left(H_{6}\right)_{0},\left(H_{6}\right)_{0}^{\theta}\right\rangle$.

As before, the vertices of $\Delta_{6}$ can all be represented by $\mathbb{Z}$-lattices. This time $\left[\Lambda_{i}\right]=\left[\Gamma_{i} \otimes_{\mathbb{Z}} \mathbb{Z}_{3}\right]$ for $i=1,2,3$, with $\Gamma_{0}:=\left\langle z_{1}, \ldots, z_{6}\right\rangle_{\mathbb{Z}}, \Gamma_{2}:=\Gamma_{0}^{\theta}$, and $\Gamma_{1}:=\left\langle z_{1}, z_{3}\right\rangle_{\mathbb{Z}} \perp\left\langle z_{5}, z_{6}\right\rangle_{\mathbb{Z}} \perp\left\langle z_{2}, z_{*}\right\rangle_{\mathbb{Z}}$, where $-z_{*}=z_{1}+2 z_{2}+2 z_{3}+3 z_{4}+$ $2 z_{5}+z_{6}$ is the highest root in the $E_{6}$ root system generated by $z_{1}, z_{2}, z_{3}, z_{4}$, 
$z_{5}, z_{6}[$ Bo, p. 268],

so that the following relations hold.

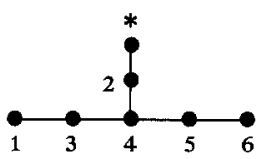

Note that $\Gamma_{1} / 3 \Gamma_{0}$ is the totally singular 2-space of $\bar{\Gamma}_{0}=\Gamma_{0} / 3 \Gamma_{0}$ spanned by $z_{5}-z_{6}+3 \Gamma_{0}$ and $z_{1}-z_{3}+z_{5}-z_{6}+3 \Gamma_{0}$, while $\Gamma_{2} / 3 \Gamma_{0}$ is the totally singular 3-space spanned by $z_{5}-z_{6}+3 \Gamma_{0}, z_{2}+z_{3}+z_{5}+3 \Gamma_{0}$ and $z_{1}-z_{3}+$ $z_{5}-z_{6}+3 \Gamma_{0}$. Moreover, we see that the stabilizer $\left(H_{6}\right)_{1}$ of the vertex $\left[\Lambda_{1}\right]$ has the form $\left(3^{3} \times 2^{3}\right) 2^{3} S_{3}$. Note that $\left(H_{6}\right)_{0} \cap\left(H_{6}\right)_{1}=2 \times\left(3^{3}\left(2 \times S_{4}\right)\right)$ and $\left(H_{6}\right)_{1}=\left[\left(H_{6}\right)_{0} \cap\left(H_{6}\right)_{1}\right]\left[\left(H_{6}\right)_{2} \cap\left(H_{6}\right)_{1}\right]$.

There is another interesting graph automorphism $\varphi$. First, let $t$ be the following product of reflections lying in $W\left(E_{6}\right): t=r_{1} r_{3} \cdot r_{5} r_{6} \cdot r_{2} r_{*}$ (where $r_{i}$ denotes the reflection in $\left.z_{i}^{\perp}\right)$.Then $t$ lies in the center of a Sylow 3-subgroup of $W\left(E_{6}\right)$ (namely, $\langle t\rangle$ is the long root group of $\mathrm{O}(5,3)$ such that $\left[\bar{\Gamma}_{0} / \bar{\Gamma}_{0}^{\perp},\langle t\rangle\right]$ is spanned by the projections of $z_{5}-z_{6}$ and $z_{2}+z_{3}+z_{5}$ into $\left.\bar{\Gamma}_{0} / \bar{\Gamma}_{0}^{\perp}\right)$, and $t^{2}+t+1=0$. Write $\varphi=1+2 t$. Then $\varphi^{2}=-3$, and $\left(u^{\varphi}, v^{\varphi}\right)=3(u, v)$ for any vectors $u$ and $v$. It is straightforward to check that $\Gamma_{2}=\Gamma_{0}^{\varphi}$, and that $\left(\frac{1}{3}\right) \Gamma_{1}^{\varphi}=\Gamma_{1}^{*}$, so that $\varphi$ normalizes $\left(H_{6}\right)_{1}$. Note also that $\theta$ normalizes $\left(H_{6}\right)_{1}$.

\section{Congruence subgroups}

In order to relate $G_{6}$ and $H_{6}$, and at the same time construct some additional chamber-transitive automorphism groups of the building $\Delta_{6}$ as well as to construct finite morphic images of $\Delta_{6}$, we introduce congruence subgroups of these two groups.

View $H_{6}$ as a group $\mathrm{O}\left(e_{6}, \mathbb{Q}_{3}\right) \cap \mathrm{GL}\left(6, \mathbb{Z}\left[\frac{1}{3}\right]\right)$ of matrices. For any integer $m>1$ not divisible by 3 , let $H_{6}(m)$ (the 'level $m$ congruence subgroup' of $H_{6}$ ) denote the normal subgroup consisting of those matrices $\equiv I(\bmod m)$. This is just the centralizer in $H_{6}$ of $L / m L$. Similarly, the group $G_{6}(m)$ is defined to be the centralizer in $G_{6}$ of $M / m M$, where $M:=\left\langle u_{1}, \ldots, u_{6}\right\rangle_{\mathbb{Z}[1 / 3]}$.

PROPOSITION 3. $H_{6}=H_{6}(2) \cdot\left(H_{6}\right)_{0}$, where $H_{6}(2) \cap\left(H_{6}\right)_{0}=\langle-1\rangle$. Thus, $H_{6}(2)$ acts regularly on the set of vertices of $\Delta_{6}$ of type 0 . It also acts regularly on the set of vertices of type 2.

Proof. Obviously $H_{6}$ acts on $L / 2 L$, preserving the quadratic form $\frac{1}{2}(u+2 L$, $v+2 L) \bmod 2$ induced on that $\mathrm{GF}(2)$-space with respect to which $L / 2 L$ is nondegenerate with Witt index 2 . The group $\left(H_{6}\right)_{0}$ induces $\mathrm{W}\left(E_{6}\right) \cong \mathrm{O}^{-}(6,2)$ on that space. This proves that $H_{6}=H_{6}(2) \cdot\left(H_{6}\right)_{0}$. Since it is clear that $H_{6}(2) \cap\left(H_{6}\right)_{0}=\langle-1\rangle$, this proves the desired regularity on vertices of type 0 . 
Now note that $\theta$ normalizes $H_{6}(2)$ (since $\theta$ normalizes $H_{6}$ and $L$ ). This proves the last assertion.

Note that the graph automorphism $\varphi=1+2 t$ defined at the end of Section 3 centralizes $L / 2 L$ and hence also $H_{6} / H_{6}(2)$.

We now determine the intersection $G_{6} \cap H_{6}$. Let ${ }^{\circ} G_{6}$ be the subgroup $\left\{G_{6} \cap \Omega\left(f, \mathbb{Q}_{3}\right)\right\}\langle-1,(12)\rangle$, where (12) denotes the reflection in $\left(u_{1}-u_{2}\right)^{\perp}$ acting as a transposition on $u_{1}, \ldots, u_{5}$. Then ${ }^{\circ} G_{6}$ has index 2 in $G_{6}$, and is normalized by $\theta$ (by a straightforward calculation). Write $\left({ }^{\circ} G_{6}\right)_{0}={ }^{\circ} G_{6} \cap$ $\left(G_{6}\right)_{0}$.

LEMMA 4. ${ }^{\circ} G_{6}=G_{6} \cap H_{6}=H_{6}(2) \cdot\left({ }^{\circ} G_{6}\right)_{0}$.

Proof. Abbreviate $D:=\left({ }^{\circ} G_{6}\right)_{0}$. Then $D=\mathrm{W}\left(D_{5}\right) \times\langle-1\rangle$, and $D$ is chambertransitive on $\operatorname{Star}\left(\left[\Lambda_{0}\right]\right)$. Moreover, $D<\left(H_{6}\right)_{0}$ (it is the $\mathrm{W}\left(D_{5}\right) \times\langle-1\rangle$ in $\mathrm{W}\left(E_{6}\right) \times\langle-1\rangle$ induced on the space $\left.\left\langle z_{2}, \ldots, z_{6}\right\rangle\right)$. Thus, $\left\langle D, D^{\theta}\right\rangle$ is a chambertransitive subgroup of both ${ }^{\circ} G_{6}$ and $G_{6} \cap H_{6}$, since $\theta$ normalizes both of these groups.

We already know that $H_{6}$ induces $\mathrm{O}^{-}(6,2)$ on $L / 2 L$. Since $\left\langle D, D^{\theta}\right\rangle$ induces a proper subgroup of $\mathrm{O}^{-}(6,2)$ containing a parabolic subgroup $2^{4} S_{5}$, it is contained in $H_{6}(2) D$. However, $H_{6}(2)$ is regular on the vertices of $\Delta_{6}$ of type 0 , so that no proper subgroup of $H_{6}(2) D$ containing $D$ can be chamber-transitive. Thus, $H_{6}(2) D=\left\langle D, D^{\theta}\right\rangle$.

In particular, $H_{6}(2) D \leqslant G_{6} \cap H_{6}$. In fact, $H_{6}(2) D=G_{6} \cap H_{6}$ since we have seen that $H_{6}(2) D$ is a maximal subgroup of $H_{6}$. Finally, ${ }^{\circ} G_{6}$ is a chambertransitive group with $\left({ }^{\circ} G_{6}\right)_{0}=\left(H_{6}(2) D\right)_{0}$, so that ${ }^{\circ} G_{6}=G_{6} \cap H_{6}$.

We have just constructed a chamber-transitive subgroup (namely, $G_{6} \cap H_{6}$ ) of $H_{6}$. In order to construct even smaller chamber-transitive subgroups, consider a subgroup $\mathrm{X}=2^{4} A_{5}$ or $2^{4} F_{20}$ of $G_{6} \cap H_{6}=2^{4} S_{5}$ (where $F_{20}$ denotes a Frobenius group of order 20 in $S_{5}$ : the normalizer of a Sylow 5-subgroup). Then clearly $H_{6}(2) \mathbf{X}$ is chamber-transitive on $\Delta_{6}$. Note that $H_{6}(2) \mathbf{X}=\left\langle\mathbf{X}, \mathbf{X}^{\varphi}\right\rangle$, where $\varphi$ is the graph automorphism defined at the end of Section 3. (For, since $\varphi$ centralizes $H_{6} / H_{6}(2)$ the chamber-transitive groups $H_{6}(2) \mathbf{X}$ and $\left\langle\mathbf{X}, \mathbf{X}^{\varphi}\right\rangle$ project onto the same subgroup of $H_{6} / H_{6}(2)$, so that the intersection of each with $\left(\mathrm{H}_{6}\right)_{0}$ is contained in $\mathbf{X}$.)

We now turn to the slightly more complicated case of $G_{6}$ in order to construct additional chamber-transitive groups. Let $M:=\left\langle u_{1}, \ldots, u_{6}\right\rangle_{\mathbb{Z}\left[\frac{1}{3}\right]}$, so that $G_{6}(2)$ is the centralizer in $G_{6}$ of $M / 2 M$. Here, $M / 2 M$ inherits the quadratic form $x_{1}^{2}+\cdots+x_{5}^{2}+3 x_{6}^{2} \bmod 2$, which is the square of a linear form whose kernel is the projection $\bmod 2 M$ of the sublattice $N$ of $M$ defined by $\Sigma x_{i} \equiv 0(\bmod 2)$. Moreover, $N / 2 M$ inherits the quadratic form $\frac{1}{2}\left(x_{1}^{2}+\cdots+\right.$ 
$\left.x_{5}^{2}+3 x_{6}^{2}\right) \bmod 2$, whose radical is the singular 1-space with representative $\Sigma u_{i}$ satisfying $\frac{1}{2}\left(\Sigma u_{i}, \Sigma u_{i}\right)=4 \equiv 0(\bmod 2)$. Thus, $G_{6}$ induces a subgroup $G_{6} / K$ of the orthogonal group $\mathrm{O}^{-}(4,2) \cong S_{5}$ of the resulting nonsingular 4-space (for a normal subgroup $K$ of $\left.G_{6}\right)$. Since $\left({ }^{\circ} G_{6}\right)_{0}$ has a subgroup $S_{5}$ acting faithfully on $M / 2 M$, it follows that $G_{6} / K \cong S_{5}$ and hence that $G_{6}=K \cdot\left({ }^{\circ} G_{6}\right)_{0}$.

LEMMA 5. $G_{6}=\left\{K \cap{ }^{\circ} G_{6}\right\} \cdot\left(G_{6}\right)_{0}$, where $K \cap{ }^{\circ} G_{6}=K \cap H_{6}$ contains $H_{6}(2)$ and is transitive on the vertices of type 0 .

Proof. Note that $K=\left\{K \cap{ }^{\circ} G_{6}\right\}\left\langle r_{6}\right\rangle$ and $\left(G_{6}\right)_{0}=\left({ }^{\circ} G_{6}\right)_{0}\left\langle r_{6}\right\rangle$, where $r_{6}$ denotes the reflection in $u_{6}^{\perp}$. Then $G_{6}=K \cdot\left({ }^{\circ} G_{6}\right)_{0}=\left\{K \cap{ }^{\circ} G_{6}\right\} \cdot\left(G_{6}\right)_{0}$.

It remains only to show that $K \cap{ }^{\circ} G_{6}$ contains $H_{6}(2)$. First note that $2(L+M)=\left\langle\Sigma u_{i}\right\rangle+2 M$ and $(L \cap M)+2 M=N$, in view of the definition of the $z_{i}$. Thus, $H_{6}(2)$ centralizes $N /\left(\left\langle\Sigma u_{i}\right\rangle+2 M\right)$, and hence lies in $K$. We already know that it lies in ${ }^{\circ} G_{6}$.

We can now construct several groups acting chamber-transitively on $\Delta_{6}$. Let $Y$ denote any of the following subgroups of $\left(G_{6}\right)_{0}=2^{6} S_{5}$, each containing-1:

$2^{5} S_{5}$ (two classes, one of which is $\left({ }^{\circ} G_{6}\right)_{0}$ and hence contains the reflection (12), while the other contains $(12) r_{6}$ );

$2^{5} A_{5}$ (this is inside $\left({ }^{\circ} G_{6}\right)_{0}$ );

$2^{6} A_{5}$;

$2^{6} F_{20}$

$2^{5} F_{20}$ (two classes, one of which lies in $\left({ }^{\circ} G_{6}\right)_{0}$ ).

Then $\left\{K \cap{ }^{\circ} G_{6}\right\} \cdot Y$ is chamber-transitive on $\Delta_{6}$. Of course, some of these chamber-transitive groups were already encountered earlier, since $H_{6}(2) \leqslant$ $K \cap{ }^{\circ} G_{6}$.

Next, let $m$ be an integer $>2$ not divisible by 3 . Then $\Delta_{6} / G_{6}(m)$ is a finite morphic image of $\Delta_{6}$ (cf. [15]), and is a geometry (by [1]) with chambertransitive group $G_{6} / G_{6}(m)$ and diagram $\Longrightarrow$. If $m=p$ is a prime $>3$ then $G_{6} / G_{6}(p)$ is a subgroup of $\mathrm{O}^{ \pm}(6, p)$ properly containing $\Omega^{ \pm}(6, p)$ (by [16], since the group induced on the vector space $M / p M$ is irreducible). The sign \pm is + if and only if -3 is a square in $\mathrm{GF}(p)$, i.e., if and only if $p \equiv 1(\bmod 3)$.

Similar remarks hold for $H_{6} / H_{6}(m)$ as well - and, for that matter, for each of the chamber-transitive automorphism groups of $\Delta_{6}$ obtained above. However, all of the resulting finite quotients of $\Delta_{6}$ are isomorphic for a given $m$. Namely, each inclusion map between two of these groups induces an isomorphism of finite geometries.

Incidentally, $G_{6} / G_{6}(2) \cong 2^{4} S_{5}$. Namely, we saw above that $G_{6} / G_{6}(2)$ has $S_{5}$ as a homomorphic image. Also, $\theta$ acts nontrivially on $N / 2 M$. From this it follows readily that $\langle\theta\rangle G_{6} / G_{6}(2) \cong 2^{5} S_{5}$. 
Using the spinor norm, it is easy to see that $G_{6}^{\prime}=\left\langle\left(G_{6}\right)_{0},\left(G_{6}\right)_{0}^{\theta}\right\rangle^{\prime}$ is the chamber-transitive group generated by $\left(\left(G_{6}\right)_{0}\right)^{\prime}$ (isomorphic to $2^{4} A_{5}$ ) and its image under $\theta$. In particular, $G_{6}^{\prime}$ is the intersection of $G_{6}$ with the kernel of the restriction of the spinor norm to $\operatorname{SO}\left(f, \mathbb{Q}_{3}\right)$. By [5], every finite homomorphic image of $G_{6}^{\prime}$ is a homomorphic image of some group $G_{6}^{\prime} / G_{6}^{\prime}(m)$. Thus, all finite quotients of $\Delta_{6}$ on which $G_{6}^{\prime}$ induces a chamber-transitive group are obtained by factoring out suitable normal subgroups of $G_{6}^{\prime} / G_{6}^{\prime}(m)$. Similar statements hold if $H_{6}^{\prime}$ is used in place of $G_{6}^{\prime}$.

Finally, we note that the group $\left\langle G_{6}, H_{6}\right\rangle$ is chamber-transitive on $\Delta_{6}$ but is not discrete. For, neither group $G_{6}, H_{6}$ contains the other; and $\mathrm{W}\left(E_{6}\right)$ is a maximal finite subgroup of $\mathrm{O}\left(f_{6}, \mathbb{Q}\right)$ generated by reflections. In fact, the group $\left\langle\left(H_{6}\right)_{0}, r_{6}\right\rangle$, which stabilizes $\left[\Lambda_{0}\right]$, is known to contain $\mathrm{O}\left(e_{6}, \mathbb{Z}\left[\frac{1}{2}\right]\right)^{\prime}$ as a normal subgroup [7].

\section{THE 5-DIMENSIONAL DISCRETE GROUPS}

In this section we will briefly sketch results analogous to those of Sections 3 and 4 for the 5-dimensional subspace $V_{5}:=\left\langle u_{1}, u_{2}, u_{3}, u_{4}, u_{5}\right\rangle$ of the space $V_{6}$ studied in those sections. Note that $V^{\prime}:=V_{5} \otimes_{\mathbb{Q}} \mathbb{Q}_{3}$ is spanned by the vectors $e_{1}, e_{2}, f_{1}, f_{2}, u_{5}$.

Let $f^{\prime}$ denote the restriction of $f$ to $V_{5}$ or $V^{\prime}$. The corresponding affine building $\Delta_{5}$ of $\Omega\left(f^{\prime}, \mathbb{Q}_{3}\right)$ is defined in Section 2 .

This time we will use the group $G_{5}$ consisting of all elements of $\mathrm{O}\left(f^{\prime}, \mathbb{Q}_{3}\right) \cap$ $\mathrm{GL}(5, \mathbb{Z}[1 / 3])$ having spinor norm in $\{ \pm 1\}\left(\mathbb{Q}_{3}\right)^{2}$, where matrices are written with respect to the $u_{i}$. As in Section 2, let $\Lambda_{0}^{\prime}=\left\langle e_{1}, e_{2}, f_{1}, f_{2}, u_{5}\right\rangle_{\mathbb{Z}_{3}}$, and note that $\Lambda_{0}^{\prime}=\left\langle u_{1}, u_{2}, u_{3}, u_{4}, u_{5}\right\rangle_{\mathbb{Z}_{3}}$. Let $r$ denote the reflection in $\left(u_{1}+u_{2}+u_{3}\right)^{\perp}=$ $\left(e_{1}-3 f_{1}\right)^{\perp}$.

Precisely as in Section 3, we find that $G_{5}$ is discrete and chamber-transitive on $\Delta_{5}$, and the stabilizer $\left(G_{5}\right)_{0}$ of $\left[\Lambda_{0}^{\prime}\right]$ is the monomial group $2^{5} S_{5}$. Moreover $G_{5}=\left\langle\left(G_{5}\right)_{0},\left(G_{5}\right)_{0}^{r}\right\rangle$.

We have $\left[\Lambda_{i}^{\prime}\right]=\left[\Sigma_{i}^{\prime} \otimes_{\mathbb{Z}} \mathbb{Z}_{3}\right]$ for $i=1,2,3$, where

$$
\Sigma_{0}^{\prime}:=\left\langle u_{1}, \ldots, u_{5}\right\rangle_{\mathbb{Z}}, \Sigma_{2}^{\prime}:=\Sigma_{0}^{\prime r},
$$

and

$$
\begin{aligned}
\Sigma_{1}^{\prime}:= & \left\langle u_{1}+u_{2}+u_{3}\right\rangle_{\mathbb{Z}} \perp\left\langle u_{1}-u_{2}+u_{4}\right\rangle_{\mathbb{Z}} \perp\left\langle u_{1}-u_{3}-u_{4}\right\rangle_{\mathbb{Z}} \perp \\
& \perp\left\langle u_{2}-u_{3}+u_{4}\right\rangle_{\mathbb{Z}} \perp\left\langle 3 u_{5}\right\rangle_{\mathbb{Z}} .
\end{aligned}
$$

Here, $\left(\Sigma_{0}^{\prime}+\Sigma_{2}^{\prime}\right) / 3 \Sigma_{0}^{\prime}$ is the totally singular 1 -space of $\Sigma_{0}^{\prime} / 3 \Sigma_{0}^{\prime}$ spanned by $u_{1}+u_{2}+u_{3}+3 \Sigma_{0}^{\prime}$, while $\Sigma_{1}^{\prime} / 3 \Sigma_{0}^{\prime}$ is the totally singular 2 -space spanned by 
$u_{1}+u_{2}+u_{3}+3 \Sigma_{0}^{\prime}$ and $u_{1}-u_{2}+u_{4}+3 \Sigma_{0}^{\prime}$. Moreover, $\left(G_{5}\right)_{1} \cong\left(2^{3} S_{4}\right) \times 2$, while $\left(G_{5}\right)_{0} \cap\left(G_{5}\right)_{1}=G L(2,3) \times 2$ and $\left(G_{5}\right)_{1}=\left[\left(G_{5}\right)_{0} \cap\left(G_{5}\right)_{1}\right]\left[\left(G_{5}\right)_{2} \cap\left(G_{5}\right)_{1}\right]$. Since $r$ fixes $\Sigma_{1}^{\prime}$ it normalizes $\left(G_{5}\right)_{1}$.

Let $L^{\prime}=\left\langle u_{1}, \ldots, u_{5}\right\rangle_{\mathbb{Z}[1 / 3]}$. Then $G_{5}$ acts on $L^{\prime} / 2 L^{\prime}$, and hence also acts on the kernel $N^{\prime}$ of the linear form $\left(u+2 L^{\prime}, u+2 L^{\prime}\right) \bmod 2$. The quadratic form $\frac{1}{2}\left(u+2 L^{\prime}, u+2 L^{\prime}\right) \bmod 2$ induced on $N^{\prime}$ is nondegenerate, with Witt index 1 and orthogonal group $\mathrm{O}^{-}(4,2) \cong S_{5}$ induced by $\left(G_{5}\right)_{0}$. It follows that $G_{5}=G_{5}(2) \cdot\left(G_{5}\right)_{0}$, where $E:=G_{5}(2) \cap\left(G_{5}\right)_{0}$ consists of $2^{5}$ diagonal matrices. If $\mathbf{X}=A_{5}$ or $F_{20}<S_{5}$ then $G_{5}(2) \mathbf{X}$ is chamber-transitive on $\Delta_{5}$. Since $r \equiv \mathbf{I}$ $(\bmod 2)$ it centralizes $G_{5} / G_{5}(2)$, and hence $G_{5}(2) \mathbf{X}=\left\langle\mathrm{EX},(\mathrm{EX})^{r}\right\rangle$. Note that $G_{5}^{\prime} \times\langle-1\rangle=G_{5}(2)\left(\left(G_{5}\right)_{0}\right)^{\prime}=G_{5}(2) A_{5}$.

This time $\Delta_{5} / G_{5}(m)$ is a finite geometry with chamber-transitive automorphism group $G_{5} / G_{5}(m)$ and diagram $\Longleftrightarrow$ whenever $m>2$ is not divisible by 3. If $m=p$ is prime, then $G_{5} / G_{5}(p) \cong \mathrm{O}(5, p)$. (Namely, (12) $\in G_{5}$, so that [16] applies.) Once again, by [5] this produces all finite quotients of $\Delta_{5}$ on which $G_{5}^{\prime}$ induces a chamber-transitive group.

\section{REFERENCES}

1. Aschbacher, M., 'Flag Structures on Tits Geometries', Geom. Dedicata 14 (1983), 21-32.

2. Bourbaki, N., Groupes et algèbres de Lie, Chap. IV, V, VI, Hermann, Paris, 1968.

3. Bruhat, F. and Tits, J., 'Groupes réductifs sur un corps local, I. Données radicielles valuées', Publ. Math. I.H.E.S. 41 (1972), 5-251.

4. Kantor, W. M., 'Some Exceptional 2-Adic Buildings', J. Algebra 92 (1985), 208-223.

5. Kneser, M., 'Normalteiler ganzzahliger Spingruppen', J. reine angew. Math. 311/312 (1979), 191-214.

6. Kantor, W. M., Liebler, R. A. and Tits, J., 'On Discrete Chamber-Transitive Automorphism Groups of Affine Buildings', Bull. AMS 16 (1987) 129-133.

7. Meixner, T., 'Klassische Tits Kammersysteme mit einer transitiven Automorphismengruppe', Habilitationsschrift, Mitt. Math. Sem. Giessen 174 (1986).

8. Meixner, T., 'Parabolic Systems: the GF(3)-Case' (in preparation).

9. Meixner, T. and Wester, M., 'Some Locally Finite Buildings Derived from Kantor's 2-Adic Groups', Comm. Alg. 14 (1986), 389-410.

10. Seitz, G. M., 'Flag-Transitive Subgroups of Chevalley Groups', Ann. Math. 97 (1973), 27-56; correction (unpublished).

11. Stellmacher, B. and Timmesfeld, F. G., (in preparation).

12. Stroth, G., 'Parabolic Systems over GF(2) whose Diagrams Contain Double Bonds, I, II' (to appear).

13. Timmesfeld, F. G., 'Tits Geometries and Parabolic Systems in Finitely Generated Groups, I, II', Math. Z. 184 (1983), 337-396, 449-487.

14. Tits, J., 'Reductive Groups over Local Fields', Proc. Symp. Pure Math. 33 (1979), 29-69.

15. Tits, J., 'A Local Approach to Buildings', in The Geometric Vein. The Coxeter Festschrift, Springer, New York, Heidelberg, Berlin, 1981, pp. 519-547.

16. Wagner, A., 'Determination of Finite Primitive Reflection Groups over an Arbitrary Field of Characteristic not Two, I, II, III', Geom. Dedicata 9 (1980), 239-253; 10 (1981), 191-203, 475-523. 
17. Wester, M., 'Endliche fahnentransitive Tits-Geometrien und ihre universellen Uberlagerungen', Mitt. Math. Sem. Giessen 170 (1985), 1-143.

Authors' addresses:

W. M. Kantor,

University of Oregon,

Department of Mathematics,

Eugene, OR 97403,

U.S.A.

Thomas Meixner,

Universität Giessen,

Mathematisches Institut,

Arndtstr. 2,

D-6300 Giessen,

F.R.G.

M. Wester,

Siemens AG,

D-8000 Munich,

F.R.G.

(Received, July 28, 1987) 